\title{
Research on Basic Information Collection of Electronic Health Records
}

\author{
Guang Dong \\ School of Computer Science and Technology \\ Changchun University of Science and Technology \\ Changchun, China, 18946663778 \\ lightdg@yahoo.com.cn
}

\author{
Chunmei Liu \\ Archives \\ Beihua University \\ Jilin City, China, 18604496661 \\ lchm0809@163.com
}

\begin{abstract}
With the use of SOA, ESB and XML technology, this paper designed message-oriented middleware and data exchange adapter based on HL7 v3, XML standard. Through data migration middleware based on XML, it realized storage and derivation of user-defined XML document and completed automatic collection of basic information for resident's health archives from other systems to community medical service software system. Furthermore, it achieved integrated access of other relevant information system through data converter adapter. It packed into "service" function module, data component needed by integrate system and data exchange platform interaction, shielding the specific technology and its realization ways used by integrated system, and get connected with communication platform in standard interface ways. It ensures high efficiency of information exchange service through the use of data cache service mechanism.
\end{abstract}

Keywords- information collection; ESB; XML; middleware.

\section{INTRODUCTION}

At present, Finland, Austria, the Netherlands, UK, Canada, the United States, Australia, France and many other countries are carrying out HL7 CDA (clinical data architecture) document interaction and sharing step by step. Although different countries have their own characteristics and train of thought in the process of practice, they use XML format for the exchange of information and the documents. Meanwhile, they started to use Web Service, or build service bus of medical and health industry and try SOA technical architecture[1][2].

With the development of computer technology, database technology, computer network technology and information industry, the construction of information industry is speeding up in China, and all industries and departments have constructed their own information system.

As we are lack of community health information guidance standard at national level in the early period of development, the existing market supply or related medical and health institutions develop "community health service information management system" software themselves. But the software are inconsistent with regard of system standard, system target, system factor, system function and system structure etc., most of the community health service software can't keep up with the development needs of the community health service. Related departments such as civil administration, public security, property management, disease control, social security, women's, children's health care, etc., designed their software according to their respective needs, thus information software can't interact between departments, and personal basic material can't be shared, which leads to the waste of resources.

Many companies in China are carrying out the research and development of new community health information management platform, for example, the community health solutions of UF, cool Bo, Neusoft. But they all started from the application requirement at various stages, and did not consider collection archiving of the basic personal data and information in electronic health records (hereinafter referred to as EHR) from other information systems[3].

As far as the practice of EHR in foreign countries is concerned, regardless of how the region is divided, its purpose is nothing but providing convenient, efficient and high-quality health services for the residents within the area. In the process of its construction, industry standards and the use of existing mature technologies, such as HL7 v3 RIM Reference Information Model, SOA integration should be used for reference more and more[4].

This paper uses the advanced EHR basic information collection technology, integrates data and information relevant basic information of health records from information systems of civil affairs, public security, property, disease control, social security, women-and-children's health care to complete the acquisition of basic information of EHR.

By providing a middleware with general, efficient and secure EHR basic personal information between community health service centers and external systems, problems such as basic personal information can't be shared, the information technology sector can't interact, information silos exist in a large number of systems and the problem of dead-files can be solved.

\section{SYSTEM DESIGN}

According to health records basic architecture and data standards of china, health records are divided into three primary categories: basic information, public health and medical services, and public health is divided into four secondary categories: children's health, women's health, disease control, disease management. 
According to the basic concepts and system architecture of health records, the basic content of health records is mainly composed of two parts: personal basic information and main health service records.

Basic personal information includes basic information such as demographic and socio-economics, as well as basic health information, some of which reflect the inherent characteristics of an individual throughout his or her life course and are relatively stable, strongly objective[5]. Mainly includes:

(1) Demographic information: such as name, sex, date of birth, place of birth, nationality, ethnicity, identity documents, educational level, marital status, etc.

(2) Socioeconomic information: such as household registration, contact address, contact information, job categories, work units, etc.

(3) Information of relatives: the number of children, parents' names, etc.

(4) Social security information: such as the category of medical insurance, medical insurance number, disability card number, etc.

(5) Basic health information: such as blood type, allergic history, vaccination history, past medical history, family genetic history, risk factors for health, disability, family health situation, etc.

(6) Filing information: such as filing date, file management agencies, etc.

EHR basic personal information collection system is a large and complex system, involving coherent work of different sides and the main content of which is obtaining basic personal information from other related systems to build up EHR. The system architecture is shown in Figure 1.

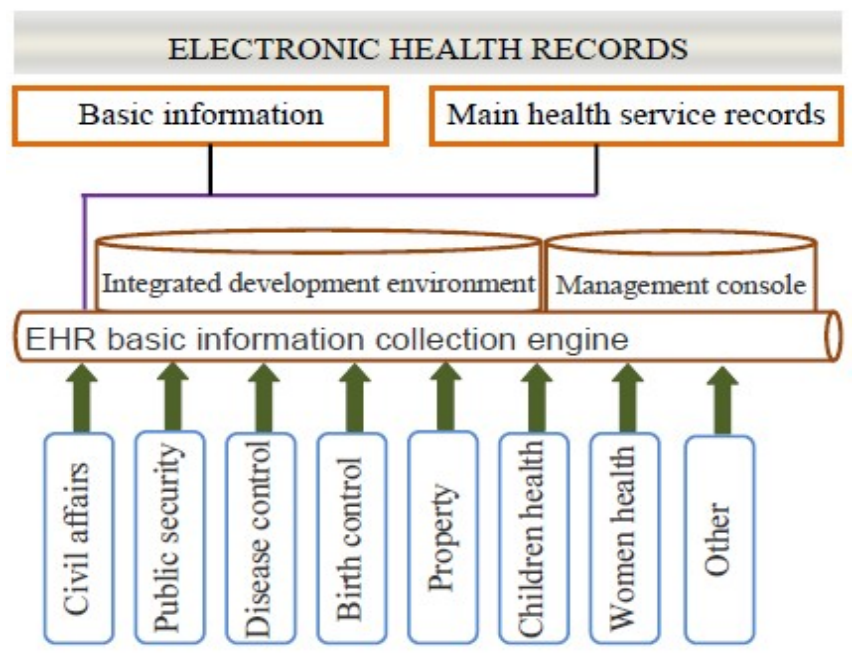

Figure 1. The structure of date collection system

Data collection system is composed of three parts: integrated development environment, the management console and the back-end engine. Integrated development environment is the creation interface of communication interface. Development interface is simple and intuitive, and the operation of its development process can mostly be completed by dragging and it supports Windows operating environment. Management Console can carry out real-time monitor and management of information at any place through browsers such as Internet Explorer. Back-office integration engine, the core of which is of Java32 \& 64, can be installed in a variety of operating systems to ensure efficient and stable operation of the system in different environments.

Data collection system, according to pre-loaded configuration file, gets the message from an external system through a variety of protocols and ways, and processes information (calibration, filtering, mapping, etc.) obtained through predefined message format and message types, and send message to the target system. Date collection workflow is shown in Figure 2.

System information collection functions need to interact with external systems. Customize interaction object according to the basic personal information domain and the relevance of external systems to collect related information. Related information will be firstly changed into HL7 standard data, and then transferred to the health records port. All collected information will be integrated to make basic personal information, and then formatted for archiving.

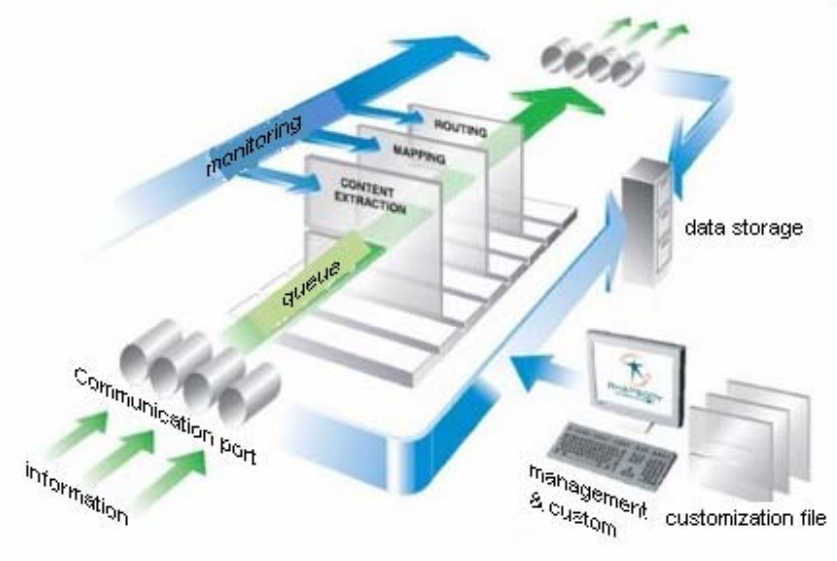

Figure 2. Date collection workflow

\section{KEY TECHNOLOGY}

\section{A. Application of XML in the acquisition and exchange of EHR basic information}

To carry out data exchange between heterogeneous databases using XML document, data exchange between the database and XML documents must be realized. Data to be exchanged will firstly be converted into XML document through XML document converter, and then be exported from the database. After parsing processing such as resolving, validation, etc., call appropriate data template through XML document template. Then convert into corresponding database data by the data converter, and import them into target database.

Therefore, on the basis of the analysis of XML and heterogeneous databases, data exchange model between 
heterogeneous databases is designed, with the use of three-tier architecture model, as shown in Figure 3. In this model, the database layer represents heterogeneous database. Data processing layer realizes the processing of source data and converting them into target data. The interface layer realizes the communications between database layer and data processing layer. Real line interface 1 , Interface $2, \ldots$, interface $\mathrm{n}$ shows the export of data from the source database and transmitted to the data processing layer. The dotted lines show the import of processed data into the target database.

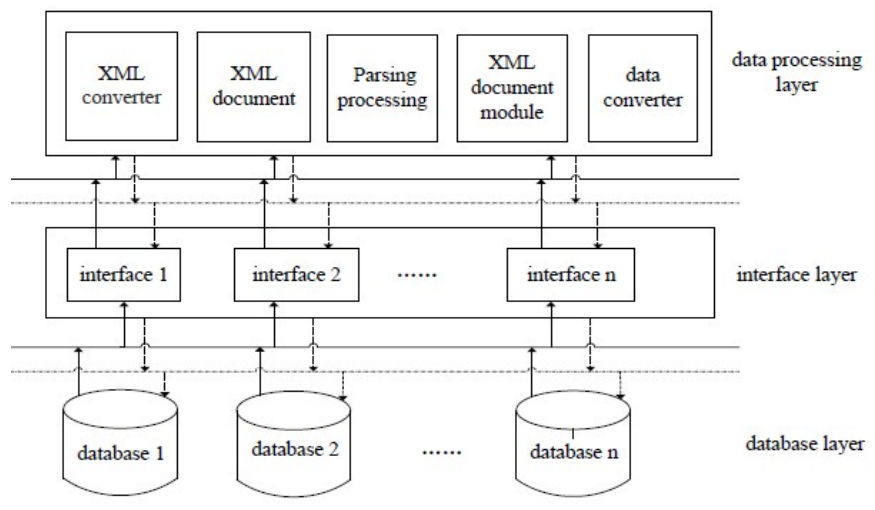

Figure 3. Data exchange module based on XML

The basic principle of this model is as following: the source database exports the data to be converted to the data processing layer through its interface. XML converter converts the data into XML document, then parsing and processing. After this, find the corresponding template in the XML document template, and convert into target database data through the data converter. Then import them to the target database through target database interface. This makes it unnecessary to consider different interface implementations. It can improve dataexchange efficiency, system scalability, and data exchange security.

\section{B. ESB technology}

ESB (Enterprise Service Bus) is a set of infrastructure realized with middleware technology and supports SOA (Service-Oriented Architecture, a service-oriented architecture), and supports services, information in a heterogeneous environment and event-based interact. Furthermore, it enjoys appropriate level of service and manageability [6]. Its working technical principle is shown in Figure 4.

Integrate workflow services on the basis of the technology of message middleware and routing function of message content. Realize integrate access to other relevant information systems through data exchange adapter. Pack into "service" function module, data component needed by integrate system and data exchange platform interaction, shield the specific technology and its realization ways used by integrated system, and then get connected with communication platform in standard interface ways[7].

Health information exchange standards HL7 (Health Level Seven) took system interconnection model OSI (Open System Interconnect) for reference. As the 7th layer protocol of the seven-layer protocol (application layer), HL7 specified grassroots organizations to adapt to the latest information. It used Web Services and XML for information exchange, thus enhanced information services and exchange capabilities based on internet. [5][8].

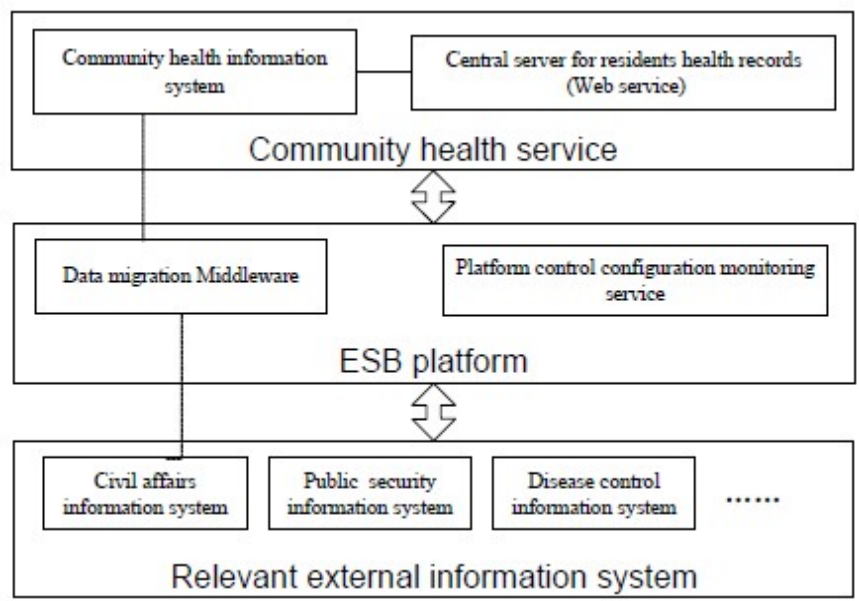

Figure 4. Model of Community health information system interacting with other systems

Data exchange adapter based on HL7 is composed of adapter core and API (Application Programming Interface). The adapter core realizes the exchange of information at information exchange center and realizes real-time monitoring of HL7 message and provides the function of distributing information application systems. API provides a set of standard interfaces for application. It is with sufficient scalability, and can be embedded into business processes and isolate communications configuration definitions and business code unrelated to business. Use middleware technology to achieve ESB infrastructure, support interaction of services and message in heterogeneous environment based on time. Furthermore, it is with the appropriate level of service and manageability.

Its key technology includes: (1) Middleware technology based on information, (2) Routing capabilities based on message content and integrated workflow services, (3) Message exchange conforms to the XML standard, compatible with a wide range of international standard protocols, such as HL7, by protocol converter.

In the overall architecture of the message bus system, various business systems send and receive business data through an adapter connected to the message exchange platform.

Heterogeneous data HL7 standardization firstly converts the data of each system to standard HL7 message format, and sent them to the receiving system in accordance with agreed communication rules. The recipient resolves the HL7 message received, and converts them into the applicable data, thus realizes the exchange of data between systems.

XML based data migration middleware, through the storage and export of self-defined XML document, fulfills the automatic collection of EHR basic information from other systems to community health services software system. 


\section{CONCLUSION}

In this paper, advanced health records building technology and information collection techniques were raised. The system can be used in community health service centers. While building EHR, basic personal information can be collected through systems as civil affairs, public security, property, disease control, social security, women and child health etc. It bears the characteristics of the one-time deployment and automatic transmission. Furthermore, it provides a common, efficient and secure EHR basic personal data and information collection system between community health service centers and relevant departments.

The paper solved many problems, such as basic information of residents cannot be shared, information technology departments cannot interact, information silos exist in a large number of systems and dead file, etc. It will promote the development of information technology in public health services, raise our community health management level, while drive the development of related industries.

\section{ACKNOWLEDGMENT}

Here, we give thanks to our professions who gave us support and advice, especially Professor HuaMin Yang in
School of Computer Science and Technology of Changchun University of Science and Technology.

\section{REFERENCES}

[1] Dong Yi-jun, "Focusing on Electronic Health Record", JOURNAL OF MEDICAL IN FORMATICS, vol.31, pp. 5-9, 2010.

[2] CAO Mao-cheng and $\mathrm{HE} \mathrm{Ji-fu,} \mathrm{"Research} \mathrm{and} \mathrm{Exploration} \mathrm{of}$ Community Health Informatization", Chinese Medical Equipment Journal, vol. 32, pp. 42-46, 2011.

[3] Dai Tao, "Development of Medical Informatics and Thinking", JOURNAL OF MEDICAL IN FORMATIC, vol. 32, pp. 2-16, 2011.

[4] LU Yun, WANG Dan, ZHAI Hong, ZHANG Xi-yu, ZHANG Yan-ling and SUN Xiao-lin, "Overview of Realization Mode for Regional Health based on Electronic Health Records", vol.27, pp. 54-56, 2012.

[5] Chen Gang, Bai Shang-wang, Dang Wei-chao and Yang Xiao-qin, "Research on Information Platform of Region Hygienism Based on Standard of HL7”, Applied Technology, vol.6, pp. 88-91, 2010.

[6] SHI Li-juan, REN Gaun-hua, DONG Lian-xu and CHEN Huang, "Research on Current Status of Health Information Related Standards", JOURNAL OF MEDICAL IN FORMATICS, vol.32, pp. 9-18, 2011.

[7] DONG, Weili SHI, Jing LIU and Yinlong Hu, "Research on the Intercommunication of Healthy Records and Hospital Medical Records", Electrical Review, vol.88, pp. 26-28, 2012.

[8] WU Xin-Dong, YE Ming-Quan, HU Dong-Hui, WU Gong-Qing, HU Xue-Gang and WANG Hao, "Pervasive Medical Information Management and Services: Key Techniques and Challenges”, CHINESE JOURNAL OF COMPUTERS, vol. 35, pp. 827-845, 2012. 